\section{SNP: A system for nonadaptive procedures*}

\author{
WALT JESTEADT $\uparrow$ \\ University of Pittsburgh, Pittsburgh, Pennsylvania 15213
}

A software system which is currently being used to collect and analyze data for a wide range of auditory psychophysical procedures is described. Its principal advantages are its scope, its ease of use during data collection, the uniformity it lends to data collection procedures in widely different experiments, and the fact that no additional programming is required to run most experiments. Although the system reflects a specific interface configuration and the availability of an 8K PDP-15, the programming has been done in FORTRAN and contains a number of features which may be of interest to even Es building hard-wired programming devices.

The basic assumptions made in designing the software system to be described here were that (1) all nonadaptive psychophysical procedures used in audition had enough in common to be run by a common data collection program, (2) the resulting data in many cases could be analyzed by a common data processing program, and (3) the investment in writing sufficiently general programs would pay off in terms of less programming later, fewer programs that Es would have to learn how to operate, and greater efficiency and uniformity in data analysis. This approach is somewhat different from the specialized language approach described by McLean (1969) and Creelman (1971). Although the data collection program could be considered as an interpreter written in FORTRAN, SNP is not a language. It cannot run all possible experiments, but those that it can run, it runs with a minimum of additional programming, in many cases none at all. Trading flexibility for a reduction in programming may be an optimum approach for many relatively specialized laboratories.

The SNP data collection program is capable of running all of the nonadaptive paradigms normally used in auditory psychophysics, with the possible exception of magnitude estimation. These include yes-no, forced choice (up to six intervals), ABX, matching category rating, absolute judgment, and various memory or information processing paradigms. The timing within a trial can be specified in terms of as many as 12 durations that can vary

* Development of the progxams discussed here was supported by NIH Grants 07790 , 04105 , and 3032 to Robert $C$. Bilger, and by Eye and Ear Hospital, the Edwards-Lazear Foundation, and the Richard King Mellon Charitable Trusts.

†Address: Bioacoustics Laboratory, Eye and Ear Hospital and School of Medicine, University of Pittsburgh, Pittsburgh, Pennsylvania 15213 . independently from $1 \mathrm{msec}$ to $10 \mathrm{sec}$. Up to 7 parameters of a trial can change from one trial to the next, and there is no limit to the number of values a parameter can take. Any of 70 parameters used to describe a "run" can be designated as a variable parameter. The variable parameters are generally the contents of a programmable oscillator of a programmable attenuator during a specific interval, but they can just as easily be the number of intervals to be presented in that trial, any interval duration, or the flag governing feedback presentation. The data collection program will store answers from 0 to 15 (16 categories) for as many as four Ss and, optionally, will also store reaction times in milliseconds. Blocks of trials can be of any length, and, with additional programming, it is feasible to present trials in a specific order (for studies involving probability learning, sequential dependencies, or continuous recognition memory, for example). By allowing independent specification of all the features of a psychophysical paradigm rather than providing a different fixed routine for data collection program provides almost as much flexibility as a specialized language.

This flexibility is achieved through a division of labor in which the data collection program relies on an input list for all of the information which distinguishes one experiment from another. This is the same approach used in many hard-wired programming devices which read and punch paper tape. In this case, the wiring has been replaced with software, and the paper tapes have been replaced with DECtape files, but the algorithm used to achieve flexibility in the data collection program could be used in designing a hard-wired device or in a smaller computer with paper tape.

Basically, SNP consists of (1) the data collection program whose capabilities have been described each anticipated procedure, the SNP briefly, (2) general data-processing programs, and (3) a general randomization program for preparing lists of trials. Additional programs which have been written include specialized randomization programs, a program for printing input or output lists, and $a$ program to facilitate modification and handling of these lists. The data collection program sets the pattern for the others and will be discussed first, after a brief description of the interface it was designed to control.

\section{INTERFACE HARDWARE} AND SOFTWARE

The need for a flexible data collection system and the flexibility achieved are both due to some extent to the scope of the computer interface. The interface consists of an external clock, a programmable oscillator (Krohn-Hite Model 453-2), three programmable attenuators, and four parallel $S$ consoles in separate sound-treated rooms. Each S console contains 3 indicator lights and 16 pushbuttons with integral feedback lights. The external clock consists of a 10-bit counter whose value can be set or read by the computer. The computer can select one of three clock rates -10 , 1 , or .1 msec-or it can have the counter determine the number of cycles in an input waveform. A 2-bit electronic switch register allows the selection of one of four channels to receive an output pulse while the clock is running. The most recent addition to the interface is a complex tone synthesizer that has been described by Wolf and Bilger (1972).

The interface is controlled by a 300-location machine-language subroutine which accepts pairs of arguments consisting of a device code number and the new contents of the device. All device contents can be specified in 12 bits or less. An answer retrieval subroutine returns the number of the $S$ who answered, the number of the answer, and the value of the clock counter at the time of the answer. Another machine-language subroutine is used to delay execution of the main program until the timer has timed out.

\section{THE DATA COLLECTION PROGRAM}

The most important design features of the data collection program are its use of input and output lists, its use of a parameter matrix, and its provision for two levels of output.

A list consists of two parts, a control block and data blocks. ${ }^{1}$ The control block is transferred to the output list file as soon as it is read, but all of its information is retained in memory during the entire run. The 
Table 1

General and Interval Run Parameters With Values for a 2IFC Paradigm

General Parameters

\begin{tabular}{lrlr}
\hline Feedback Flag & 5 & Number of Intervals Used & 2 \\
Feedback Duration & 1000 & Fixed Device Code 1 & 8 \\
Rise-Decay Duration & 50 & Contents & 1000 \\
Break Duration (Min) & 5 & Fixed Device Code 2 & 9 \\
Intertrial Interval & 3000 & Contents & 20 \\
\hline
\end{tabular}

Interval Parameters

Presentation Interval

\begin{tabular}{lcccccc} 
& 1 & 2 & 3 & 4 & 5 & 6 \\
\hline Off Time & 0 & 500 & - & - & - & - \\
Device Code & 8 & 8 & - & - & - & - \\
Contents & $1000 *$ & $1010 *$ & - & - & - & - \\
Device Code & - & - & - & - & - & - \\
Contents & - & - & - & - & - & - \\
Device Code & - & - & - & - & - & - \\
Contents & - & - & - & - & - & - \\
On Time & 500 & 500 & - & - & - & - \\
Coincidence Indicator Number & 2 & 2 & - & - & - & - \\
Electronic Switch Number & 1 & 1 & - & -
\end{tabular}

information includes initial values for all of the parameters which govern a run, a list of the parameters to be varied from trial to trial, the number of trials in the run, and sorting and labeling information to be used in data analysis.

Each data block contains specific information about 31 trials, and only one block of specific information is in memory at any one time. During the intertrial interval following the 31st trial, the specific information concerning these trials, including Ss' answers, is transferred to the output list file. It is then replaced by new information from the input list file. The control block variable indicating the total number of trials is used by the program to determine the number of data blocks which follow a control block. The specific information about a trial includes as many as seven parameter values to be used in running that trial. It also includes the correct answer, sorting information for the data analysis programs, and space for a 4-bit answer from each of four Ss. The format of this information is presented in Fig. 1.

Because all information in the input list is transferred to the output list, these lists are identical except for the addition of Ss' answers to the output list. This means that it is not necessary to run all four Ss at the same time. If a $S$ misses a session, the output list generated by the other Ss can be used as the input list for the missing S's make-up session. The output list generated in that session will be the same as if all four Ss had been run simultaneously.

The 70 parameters which describe a run are used in a fixed sequence of instructions in such a way that the parameter values completely determine the events which occur during a trial and between trials. The parameters take the initial values specified in the control block and then certain parameters are changed from trial to trial.

The 70 parameters are organized in a matrix consisting of six 10-parameter lists that describe the six possible presentation intervals within a trial, and one 10-parameter list that describes more general aspects of a trial or of the entire run. The parameter names and one set of possible values are presented in Table 1.

The 10 parameters for a given interval are used in two groups. The three designated devices are set during off-time. The coincidence indicator and electronic switch selection are synchronized with on-time. Because a loop is used for the six presentation intervals, the number of intervals could be expanded without increasing the size of the main program.

Five of the general parameters, the two device codes and contents, and the break duration are used only once in the run. The rise-decay duration tells the program how long it must wait after the off-pulse at the beginning of each off-time before it can safely change the contents of devices.

Before a given trial is executed, the data collection program transfers the parameter values contained in the specific information about that trial to positions in the parameter matrix. This transfer is governed by a seven-location, control-block list called MAP, which contains values from 0 to 70. Nonzero values are used as subscripts for the parameter matrix. It is unnecessary, therefore, to include space in the description of each trial for all of the different aspects of a trial that might be varied from one trial to the next in some future psychoacoustics experiment. The MAP list assigns the space available to any 7 of the 70 possible parameters.

The values in Table 1 illustrate the way a typical 2IFC procedure would be specified in terms of this system. The device code 8 designates the programmable oscillator. The asterisks next to the contents specified for this device indicate that these values are variable parameters that would be changed from trial to trial. Values in the MAP list that pointed to these positions in the parameter matrix would cause new values to be transferred to these positions from the information specific to each trial. Correct-answer feedback would also be based on the information specific to each trial.

The large blank areas in Table 1 indicate the extent to which the capabilities of the system exceed the requirements of typical psychoacoustics paradigms. Table 2 contains the parameter values required for a more demanding paradigm, a pitch-memory experiment described by Elliott (1971). Elliott's Ss listened to three pains of tones-two target tones, two intervening tones, and two comparison tones. Their task was to indicate which comparison tone differed-and in which direction-from the corresponding member of the target pair. In this example, all six frequencies vary from one trial to the next (the target frequencies shifted from trial to trial) and each S's answer can take one of four values. More information about the procedure can be found in Elliott's (1971) description of Experiment IIi.

The data collection program is also flexible in the forms of output that it provides. At the end of a run or at designated breakpoints within a run, the data collection program stops and prints, on the Teletype, summary data for each $\mathbf{S}$. More detailed summaries can be created with the general data analysis program using the trial-by-trial data stored in the output list, but the summary that is immediately available contains as much information as most hard-wired devices with counters provide. The summary consists of the number of correct and incorrect responses associated with each response button for as many as eight buttons. If more than eight response categories are used, the program sums across categories and prints the overall percent correct. The summary is printed in terms of responses rather than stimuli because the number of possible responses is more limited, differences between responses are more easily defined to the program than differences between stimuli, and the response information is better for 
determining the appropriateness of the $S^{\prime}$ ' use of categories in confidence rating experiments. The summary also contains the mean reaction time in milliseconds for each $\mathbf{S}$ across all the trial in the list.

The data collection program can easily be operated by an undertraduate who is also $\mathbf{8}$ in the experiment. Only three questions mut be anwered from the Teletype-the number of S8, the input-list file name, and the output-lint file nume. After the information hos been entered, the data collection program lifhts the anvwer indientor on the 83' consoles and waits for one anmer an indication that everyone is ready for a run to begin. After printing the summary at the end of a lin, the program times a break period whose duration is specified by a control-block variable, and then it lights the answer indicators to signal the beginning of a new run. The conventions for using the program are uniform across all of the possible experiments it can run.

\section{THE DATA PROCESSING PROGRAMS}

Although the data collection program sets the pattern for all SNP programs, the system also has been designed with flexible data processing in mind. The information required for this has been included as an integral part of the trial-by-trial bulk-data files. The approach that has been taken and the flexibility that has been achieved will be illustrated in terms of a program for processing signal detection data, SDD.

SDD has been designed to meet requirements that are different from those which governed the form of the data collection program. The capabilities required include: sorting trial-by-trial data to produce 2 by 2 matrice for each subject for each condition, printing the matrices with sufficient labeling of conditions to make printouts generally interpretable, storing the matrices as data summaries on DECtape and retrieving them later, and calculating and printing the signal detection parameters. An additional requirement was sufficient flexibility in the use of input and output devices to allow control of processing from a remote Teletype, batch processing, or the use of separate output devices for questions and data summaries. Because large fixed amounts of time are required to search bulk data and print detailed summaries, only a small percentage of the total time can be saved by writing small programs that avoid swapping segments to minimize running time. Therefore, SDD has been written as a single program which calls different subroutines from DECtape to perform each option as it is selected.

Tohle 2

General and Interval Run Parmetex With Values for Memory Paradign Uned by blliott (1971)

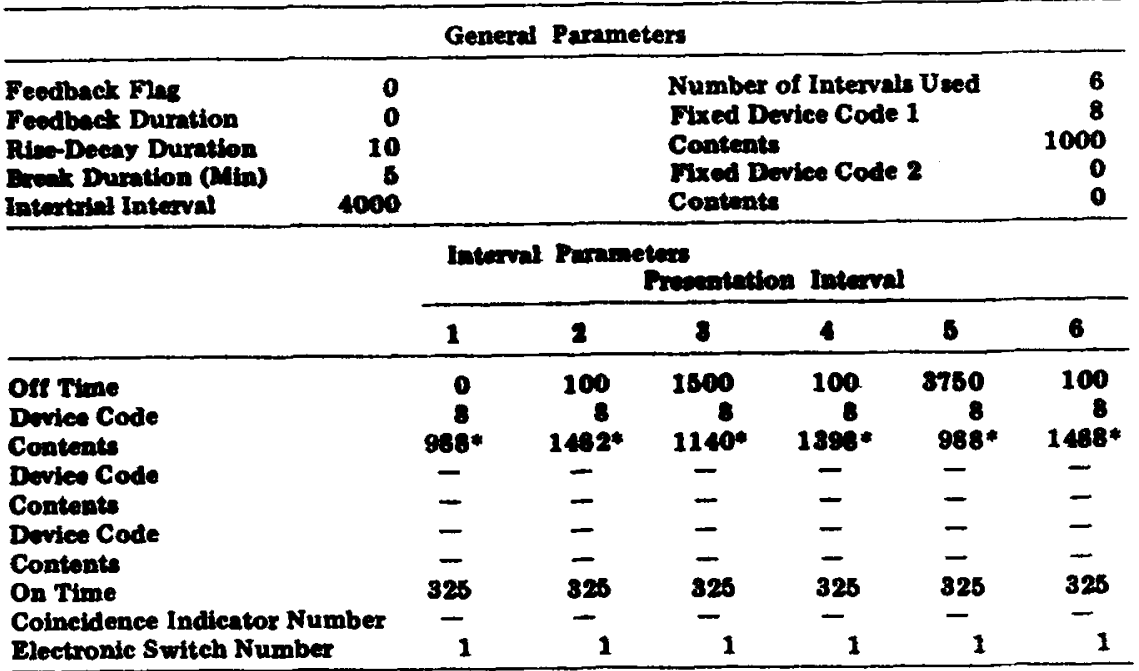

SDD resembles the data collection program in its heavy reliance on the bulk data file, rather than on the operator, for the information necessary in sorting and printing. This information is coded in five arrays in each control block and in the descriptions of each trial in the data blocks.

The most important feature in the organization of the corting and labeling information is the assumption that experiments with large number of conditions can be represented in terms of factorial designs. The advantage of making such an assumption is that, to the extent that it is true, a large number of conditions can be described by specifying the factors of the design and the possible levels of each factor that can occur. Most large experiments use sets of conditions close enough to complete factorial designs to make this the most economical approach, both for printing the descriptions of conditions and for allowing the operator to indicate conditions that should be combined or should not be included.

The control block information includes five-character names for as many as four factors, the number of levels of each factor which can occur, and space for integer labels for as many as $\mathbf{4 0}$ levels. In the extreme case, a factorial design involving 10 levels of each of four factors, the limited information in the control block can be used to generate correct labels for 10,000 conditions.

The representation of conditions in the experiment in terms of positions in a factorial design is carried over to the descriptions of individual trials. Four of the eight woirds used to describe an individual trial contain 5-bit numbers which indicate the level of that trial on each of the four possible factors of the factorial design. The location of this information is indicated in Fig. 1. Although a factor level and a parameter value are stored in different portions of the same word, there is no correspondence required between these values. Because the number of parameter values can exceed the maximum number of factors, a complete correspondence may be imporsible. The independent sorting information is provided because the parameter values do not generally lead to an efficient description of the actual experimental conditions.

Using this sorting and labeling information, SDD can compile, print, store, and analyze as many as 40 2 by 2 matrices at a time for each of four Ss. If the input lists for the data collection program have been prepared correctly, the data for experiments with no more than 40 conditions can be analyzed by combining all of the bulk-data files in a single file and using each SDD option only once. The result will be one summary file, one printout of 2 by 2 matrices, and one printout of the corresponding signal detection parameters.

When asked to compile matrices for a bulk-data file, SDD reads the first control block in the file to determine the number of conditions and the structure of the design. The operator can reduce the number of matrices compiled by indicating that levels on certain factors should be combined with other levels or that certain levels should be ignored. If the number of conditions exceeds 40, the operator can compile matrices for all of the individual conditions by having SDD ignore certain conditions one time and other conditions the next.

Given this solution of the sorting and labeling problems, the approaches taken in meeting the other requirements can be relatively straightforward. The subroutines 


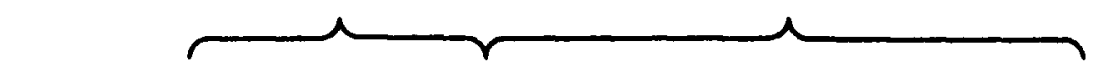

WORD I

parameter value I

WORD 2

parameter value 2

WORD 3 LEVEL ON FACTOR I I

parameter value 3

WORD 4 LEVEL ON FACTOR $?$

WORO 5 LEVEL ON FACTOR 3 I

parameter VAlue $\mathbf{s}$

WORD 6 LEVEL ON FACTOR 4.

WORD 7 CORRECT ANSWER |

parameter value 6

parameter value 7

WORD 8 | SUBJ. 4 ANSW. I SUBJ. 3 ANSW. I SUBJ. 2 ANSW. SUBJ. I ANSW I

Fig. 1. Format of the eight words used to code all of the information specific to an individual trial.

which compile the 40 matrices leave behind in core the matrices plus most of the information contained in the first-bulk data file control block. The space originally occupied by the 70-location parameter array is used for an array which indicates which factor levels were combined or ignored in compiling matrices, but all of the labeling information remains in its original form. This "working area" can be stored as a summary file on DECtape and, of course, any summary file stored previously can be loaded into the working area or matrices from two files with identical structures can be added in this area. Printing the matrices does not alter the working area in any way.

The working area is altered, however, by the subroutine which calculates signal detection parameters. ${ }^{2}$ The four values in each matrix are interpreted as areas under two normal curves (Green \& Swets, 1966) and converted to $z$ scores and corresponding ordinates using the algorithm given by Hastings (1955). In addition to calculating values of $d^{\prime}$ and $\beta$ for each matrix, the subroutine also calculates the standard error of $d^{\prime}$ using the procedure described by Gourevitch and Galanter (1967). The cell frequencies in each matrix are replaced by the signal detection parameters when they are calculated. The values of $d^{\prime}, \beta$, and $S_{d^{\prime}}$ are multiplied by 10,000 and converted to integers for this purpose. The fourth cell frequency is replaced by the total $N$ for the matrix. The parameter information can then be printed by a routine very similar to the routine that prints the cell frequencies, because the labeling information is identical, and they are stored in the same way. A flag set by the compilation and calculation routines prevents the printing of the working area by the wrong routine.

SDD can be used to analyze a large range of experiments and in each case it will provide a summary of the data that reflects the unique features of the design and the specific nature of the conditions. The format of the information in the bulk data files which gives SDD these capabilities will give the same capabilities to any other data analysis program. When such a program is written, it can be applied automatically to any bulk data for which it is appropriate.

\section{RANDOMIZATION PROGRAMS}

The burden of supplying the information required during data collection and data analysis falls on the randomization programs and on the person using these programs. Even at this stage, however, it is possible to write general programs which can create input lists for many different experiments. When special randomization programs do have to be written, standard subroutines can be used to perform the functions common to almost all randomization programs. The result is that even very different randomization programs tend to have similar operating instructions.
The nature of the programs and procedures required for generating randomization lists will be illustrated in terms of GENRAN, a program designed to create lists for any experiment that can be approximately described in terms of a factorial design. GENRAN assumes that different types of trials within a list should be equally likely and that there should be no sequential dependencies between trials.

GENRAN, like SDD, has been written as a single program which calls different subroutines from DECtape to perform each option. The options fall in three categories: inputting or modifying information about an experiment, saving the information on DECtape or retrieving it, and using the information to create a specified number of randomization lists.

All of the information which the data collection program and SDD get from the control block of the trial-by-trial list can be entered by selecting various input options. The information is stored in the order in which it must appear in the control block so that one area of core can simply be transferred to tape when lists are created. One option in GENRAN allows the operator to enter the total number of trials in the list, the number of trials between breaks, and the value to be used in initializing the random number subroutine. Another allows the operator to enter the factor names and the label for each factor level. Another option allows the operator to set all of the nonvariable parameters by specifying parameter names and values. The subroutine used for this can be used in any randomization program. The last input option allows the operator to specify the values of the variable parameters which correspond to each level on each factor. More than one parameter and more than one value of each may be involved in the specification of a single factor level. When more than one value is involved, the values are assumed to be equally likely. As was the case in SDD, the factorial design assumption allows all of the conditions to be described economically in terms of the factors underlying them.

When an experiment has been completely described, the arrays containing the information can be saved on DECtape so that all of the input does not have to be repeated when additional lists are needed. Because GENRAN options can be executed in any order, it is often possible when preparing for a new experiment, to begin by retrieving the information stored previously during preparation of a similar experiment. Then only the information which distinguishes the new experiment from the old one must be entered. A library 
of experimental procedures is soon generated in this way.

In using this information to create lists, the operator must indicate the number of lists to be created and the factors that represent variables to be randomized within lists rather than between lists. The randomization can be done with or without replacement. If it is done without replacement, GENRAN calculates a quota for the number of times any single type of trial will be allowed to occur and counts the number of times each type of trial has occurred in the list during randomization. When a specific type of trial has been randomly selected, GENRAN generates a random integer in the range from one to the quota and includes the selected trial in the list only if the random integer exceeds the value that indicates the number of times a trial of that type has already been included. Note that a quota system alone, without the additional randomization, is not equivalent to randomization without replacement.

GENRAN can be used to create individual lists for specific conditions or to create, in one session, all of the lists for a single experiment. In addition to being able to generate most lists directly, it serves as a model and $a$ starting point for special randomization programs.

\section{UTILITY PROGRAMS}

Two utility programs which contribute to the power of this system should be noted briefly. The first is a program which prints all of the information in bulk data files. Although this is not a necessary step in data analysis, it does permit the $E$ to verify that GENRAN or a special randomization program has generated a list with all of the required characteristics. In printing a well-formatted description of a bulk data file, the program uses all information in the way it would be used by later programs. A correct printout virtually insures correct data collection and analysis. The second utility program allows extensive editing of bulk data files, including modification of the parameter array or any of the text arrays. Modifications which influence only data analysis can be made even after the data have been collected. The data-file nditor is also useful in segmenting data files which contain more than one list and in transferring lists from one file to another. When an experiment requires running several different one-condition lists in a specific order, for example, it is possible to keep lists for different conditions segregated in different files, then transfer one list from each file to a smaller temportary file which will be the input file on a specific day.

\section{SUMMARY}

One approach to solving the programming problem faced by most computerized laboratories is to write a group of very general programs. The system described here has been presented as an argument in favor of this approach. It is not, of course, the best approach for all laboratories and its vaiue may depend to some extent on the nature of the facilities available. It would be more difficult to implement on a smaller computer and would not be as necessary on a computer large enough to support time-shared program preparation. The value of this approach also depends on the uniformity of programming skill among the Es using the laboratory and on the proportion of their time spent in doing research. If there are a number of full-time members of the laboratory staff who have equal amounts of time to devote to programming and to understanding the nature of the computer interface, then development of a system such as the one described here, which requires a great deal of coordination, would probably not be the optimum approach.

The specific details of the SNP programs have been presented as arguments in favor of certain design features in a general system. Because some of them could be implemented in hardware or in specific data analysis programs, they can be accepted or rejected independently of the overall argument in favor of general programs. The most critical features of SNP are the use of input and output lists, the use of a parameter matrix and map array, the representation of conditions in terms of a factorial design, and the coding of all information in the input lists. The system based on these features is flexible, yet more powerful and convenient to use than the specialized programs for each experiment that it has replaced.

\section{REFERENCES}

CREELMAN, D. D. Rapid response and flexible experimental control with a small on-line computer: PSYCLE. Behavior Research Methods \& Instrumentation. $1971,3,265-267$.

ELLIOTT, L. L. Auditory memory for one and two tones. Journal of the Acoustical Society of America, 1971, 49, 450-456.

GREEN, D. M. \& SWETS, J. A. Signal detection theory and psychophysics. New York: Wiley, 1966.

GOUREVITCH, V. \& GALANTER, E. A significance test for one parameter isosensitivity functions. Psychometrika. 1967, 32, 25-32.

HASTINGS, C. Approximations for digital computers. Princeton, N.J: Princeton University Press, 1955.

MCLEAN, R. S. PSYCHOL: A computer language for experimentation. Behavior Research Methods of Instrumentation, $1969,1,323-328$.

WOLF, R. V. \& BILGER, R. C. Generation of complex tones. Behavior Research Methods \& Instrumentation, 1972, in press.

NOTES

1. The term "block" refers to a physical unit of DECtape in which the PDP-15 system software can store as many as $\mathbf{2 4 9}$ 18-bit words. Because nonformatted FORTRAN read or write statements involve a minimum transfer of one block, tape storage is optimized by treating the lists as groups of approximately 249 values.

2. Although the system as a whole will not be distributed at this time, listings of this subroutine and details concerning other specific procedures are available upon request. 